\title{
Maîtrise de phénomènes de saut dans les réponses fréquentielles des structures avec non-linéarité localisée
}

\author{
Johan Der Hagopian ${ }^{1}$, Mohamed BelhaQ ${ }^{2}$, Zäzilia Seibold ${ }^{1}$ et Jarir Mahfoud ${ }^{1, a}$ \\ 1 Université de Lyon, CNRS, INSA-Lyon, LaMCoS UMR 5259, 69621 Villeurbanne, France \\ 2 Université Hassan II-Casablanca, Laboratoire de Mécanique, Casablanca, Maroc
}

Reçu le 27 juillet 2009, accepté le 10 juin 2010

\begin{abstract}
Résumé - Certaines structures non-linéaires sont dotées d'hystérésis responsables de phénomènes de saut dans les réponses fréquentielles et d'instabilités. Dans ce travail, on étudie ces phénomènes analytiquement, numériquement et expérimentalement sur une poutre encastrée libre dont le comportement non-linéaire est modifiable. La non-linéarité dans la structure est introduite par des actionneurs électromagnétiques. La structure ainsi que les actionneurs sont dimensionnés afin de mettre en évidence le phénomène de saut. Les résultats obtenus analytiquement en utilisant une méthode de perturbation sur un modèle non-linéaire simplifié ont été validés expérimentalement et par simulations numériques. Ces résultats montrent qu'il est possible de maîtriser le phénomène de saut dans les réponses fréquentielles en utilisant des actionneurs électromagnétiques.
\end{abstract}

Mots clés : Comportement dynamique / saut d'amplitude / actionneur électromagnétique / méthode de perturbation / validation expérimentation

\begin{abstract}
Control of the amplitude jump phenomena of structures with localized nonlinearity. Some forced nonlinear structures present hysteresis leading to jump phenomena and instabilities in the frequency response. In this paper, we investigate these phenomena analytically, numerically and experimentally on a structure whose nonlinear behaviour can be modified. The chosen structure is a free clamped beam; the non linearity is introduced by using electromagnetic actuators. The structure and the actuators are dimensioned in order to highlight this jump phenomenon. The results obtained analytically using a perturbation method on a simplified single mode model have been validated by numerical simulations and through experiments. These results show the possibility of controlling the jump phenomena in frequency forced response by acting on the electromagnetic actuators.
\end{abstract}

Key words: Dynamic behavior / amplitude jump / electromagnetic actuator / perturbation method / experimential validation

\section{Introduction}

Le progrès réalisé dans différents domaines de l'ingénierie fait que les systèmes mécaniques modernes opèrent dans des conditions où les couplages multi physiques jouent un rôle important, et font apparaître des comportements non-linéaires qu'il faut prendre en considération dès la conception. Les tendances actuelles concernent l'augmentation du rendement et de la fiabilité, la diminution des dimensions, des coûts d'exploitation

\footnotetext{
a Auteur pour correspondance:

Jarir.mahfoud@insa-lyon.fr
}

et des impacts environnementaux. Les machines doivent donc opérer dans des conditions respectant les normes de sécurité, de sûreté, de confort et d'environnement.

Le comportement non-linéaire d'un système mécanique peut se manifester sous différentes formes [1,2]. À titre d'exemple, dans le secteur automobile, les équations de mouvements des vibrations latérales d'un système d'entraînement par courroies peuvent mener à un régime oscillatoire avec des excitations paramétriques régi par les équations de Duffing [3]. Réduire l'impact de ce type de comportement nécessite une compréhension des phénomènes de base à la fois analytiquement et expérimentalement. Il est donc important de pouvoir 

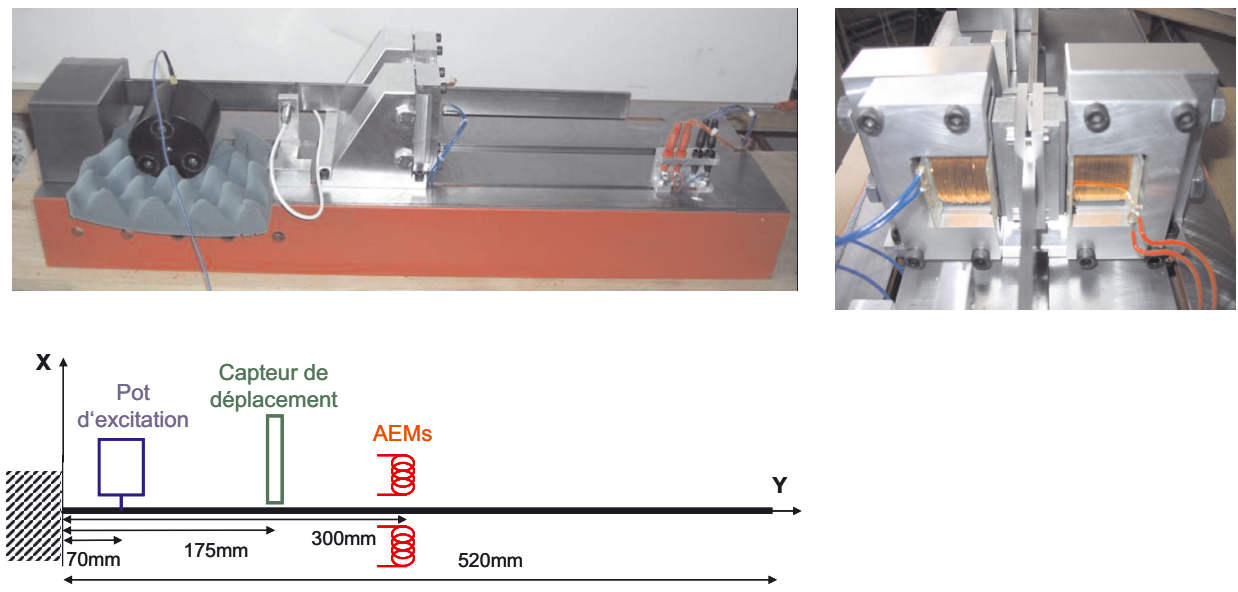

Fig. 1. Banc d'essai.

contrôler les vibrations à grandes amplitudes, de déterminer des critères de stabilité ou de supprimer l'hystérésis responsable des phénomènes de saut dans les systèmes vibratoires. Des résultats analytiques récents ont montré que l'introduction d'une excitation à haute fréquence dans un système vibratoire peut avoir un effet sur l'élimination de l'hystérésis $[4,5]$.

D'autre part, les paliers magnétiques actifs (PMA) ont montré leurs efficacités dans plusieurs applications industrielles. Ils ont l'avantage d'opérer sans contact et ils peuvent être utilisés dans des applications nécessitant une propreté absolue ou dans des milieux corrosifs [6]. Les PMA ont également l'avantage de pouvoir agir sur l'arbre directement ou indirectement en les associant à des paliers classiques, dans ce cas les PMA agissent comme des actionneurs $[7,8]$.

L'objectif de cet article est d'étudier analytiquement, expérimentalement et numériquement, l'influence des actionneurs électromagnétiques sur les phénomènes de saut d'amplitude que l'on peut rencontrer dans la réponse d'une poutre encastrée libre excitée par une force extérieure sinusoïdale. Pour ce faire, on développe dans un premier temps une étude analytique sur un modèle simplifié masse-ressort-amortisseur à un seul degré de liberté non-linéaire en utilisant une méthode de perturbation. Cette étude permet d'obtenir la courbe de réponse du système considéré dans une approche à un seul mode. Un banc de simulation simple est ensuite réalisé dont le comportement non-linéaire est pilotable, la non-linéarité étant introduite par une force générée par une ligne d'action électromagnétique composée de deux électroaimants placés de part et d'autre de la poutre et parcourus par un même courant continu d'amplitude constante. Cette force est la somme des deux forces électromagnétiques générées par les deux actionneurs : chacune dépend de l'inverse du carré du déplacement de la poutre au niveau des actionneurs.

Le papier est organisé en plusieurs sections; après la description du banc et de la ligne d'action dans la section 2, un traitement analytique approximant la courbe de réponse du système est effectué dans la section 3. Dans la section 4 , on présente les résultats obtenus par simulations numériques en utilisant une méthode des élémentsfinis. La section 5 est consacrée à l'étude expérimentale. Les conclusions sont dressées dans la dernière section.

\section{Description du banc d'essai}

Le banc d'essai (Fig. 1) est constitué d'une poutre en acier de section constante $(5 \mathrm{~mm}$ d'épaisseur et $30 \mathrm{~mm}$ de largeur) encastrée à une extrémité et libre à l'autre. La raideur non-linéaire est apportée par une ligne d'action, composée de deux actionneurs électromagnétiques, parcourus par un courant constant. L'ensemble est monté sur un bâti en acier. Le banc est conçu afin d'avoir un positionnement précis et facile de l'actionneur et de son entrefer. Dans cette étude, l'actionneur est placé à $300 \mathrm{~mm}$ de l'encastrement de sorte à avoir suffisamment d'influence et à éviter des déplacements supérieurs à la valeur de l'entrefer pour la plage fréquentielle étudiée.

Il est à noter qu'un actionneur électromagnétique (AEM) ne peut seulement générer que des forces d'attraction. Ainsi deux actionneurs de caractéristiques identiques montés de part et d'autre de la poutre et parcourus simultanément par le même courant sont nécessaires pour symétriser leurs effets sur la poutre. Chaque AEM se compose de deux assemblages de tôles ferromagnétiques isolées, le premier est en forme de (E) qui reçoit la bobine et le second en forme de (I) qui est fixé directement sur la poutre. L'entrefer nominal (e) entre ces deux assemblages est choisi suffisamment petit afin que les pertes magnétiques soient négligeables. La description géométrique des actionneurs est récapitulée sur la figure 2.

Les actionneurs sont conçus pour délivrer une force d'attraction maximale de $300 \mathrm{~N}$ pour un courant maximum admissible de $3 \mathrm{~A}$. Ils délivrent des forces d'attraction dont les amplitudes sont fonction du courant qui les parcourt. Ce courant est pilotable et peut être constant ou variable. Dans la présente étude on se limite au cas où le courant est constant. À partir des théorèmes classiques de circuits magnétiques et électromagnétiques, et 


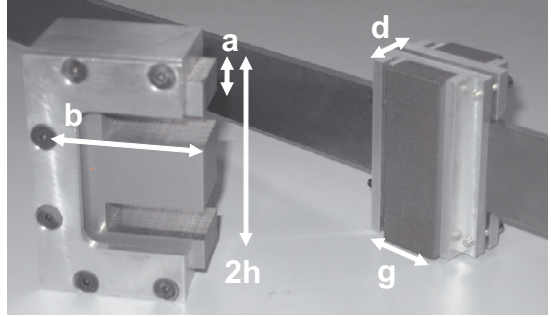

$a=10 \mathrm{~mm}$
$b=40 \mathrm{~mm}$
$\mathrm{~h}=30 \mathrm{~mm}$
$\mathrm{~d}=10 \mathrm{~mm}$
$\mathrm{~g}=21 \mathrm{~mm}$
$\mathrm{~N}=180$ spires

Fig. 2. Détails de l'actionneur électromagnétique.

en supposant un flux magnétique conservatif et de pertes négligeables, la relation qui lie la force électromagnétique $\left(F_{\mathrm{em}}\right)$ au courant $(I)$ et au déplacement, calculé ou mesuré, au droit de l'actionneur $\delta_{\mathrm{a}}(t)$ peut s'écrire sous la forme :

$$
\begin{aligned}
F_{\text {em_actionneur 1 }} & =\frac{N^{2} \mu_{0} a g I^{2}}{2\left(\left(e+\delta_{\mathrm{a}}(t)\right)+\frac{b+h+d-2 a}{\mu_{r}}\right)^{2}} \\
\text { et } \quad F_{\text {em_actionneur 2 }} & =\frac{N^{2} \mu_{0} a g I^{2}}{2\left(\left(e-\delta_{\mathrm{a}}(t)\right)+\frac{b+h+d-2 a}{\mu_{r}}\right)^{2}}
\end{aligned}
$$

Les quantités $a, b, h, d$ et $g$ correspondent aux caractéristiques géométriques des actionneurs. $\mu_{0}$ représente la perméabilité du vide $\left(4 \pi \times 10^{-7}\right.$ H.m $\left.{ }^{-1}\right), \mu_{\mathrm{r}}$ est la perméabilité relative (sans dimensions) qui dépend de l'entrefer et qui peut varier en fonction de la température. Sa valeur a été déterminée expérimentalement [9] en mesurant la force générée en fonction d'un balayage en courant (en montée et en descente) pour différentes valeurs d'entrefer (Fig. 3). Dans la plage d'utilisation du courant, la valeur moyenne de la perméabilité obtenue est de 740 c'est donc la valeur utilisée dans cette étude.

Les mesures obtenues montrent que la force varie en fonction de la valeur au carré du courant, et que les effets d'hystérésis dus au champ électromagnétique peuvent être considérés comme négligeables.

Pour la suite, et afin d'avoir une expression comparable à l'étude analytique, la force électromagnétique est mise sous la forme :

$$
F_{\mathrm{em}}=\frac{C_{1}}{\lambda^{2}}\left(\frac{1}{(1-z)^{2}}-\frac{1}{(1+z)^{2}}\right)
$$

où $C_{1}=\frac{1}{2} N^{2} \mu_{0} a g I^{2}, \quad \lambda=e+C_{2}, C_{2}=\frac{b+h+d-2 a}{\mu_{\mathrm{r}}}$ et $z=\frac{\delta_{\mathrm{a}}}{\lambda}$.

\section{3 Étude analytique}

Dans cette section, une étude analytique sur un modèle simplifié est réalisée. En effet, l'équation du mouvement d'une poutre encastrée libre à un seul mode soumise à une excitation extérieure sinusoïdale et à une force électromagnétique de courant constant, peut être réduite

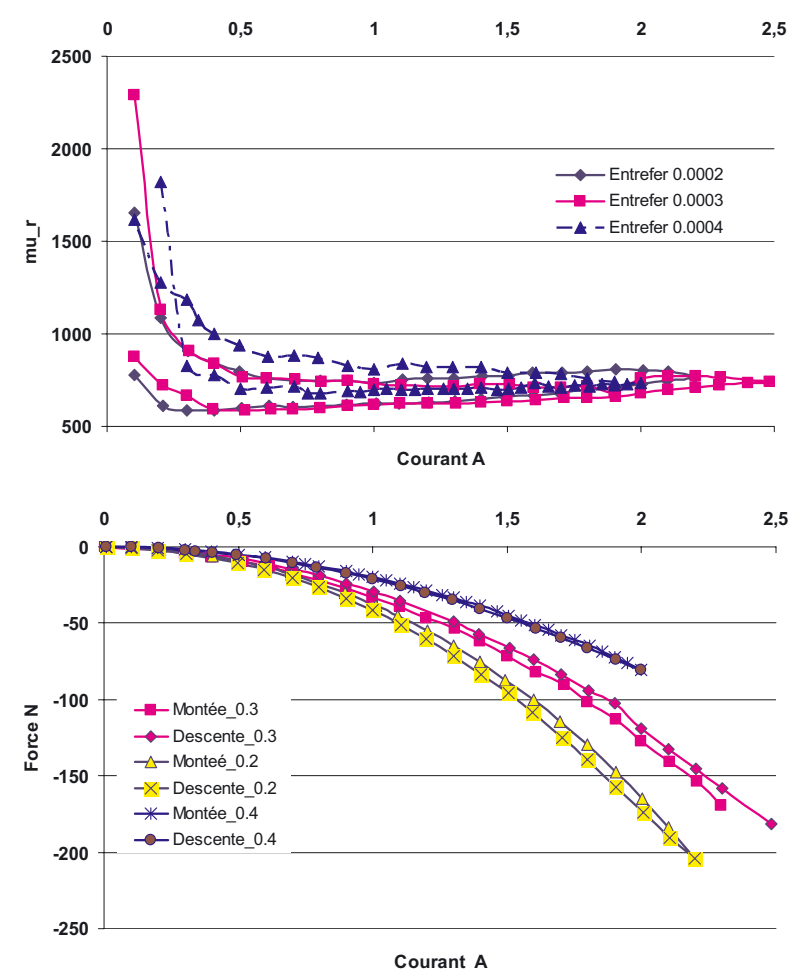

Fig. 3. Perméabilité magnétique relative et forces en fonction du courant pour différents entrefers.

à un système masse-ressort-amortisseur qui peut s'écrire sous la forme adimensionnelle :

$$
z^{\prime \prime}+c z^{\prime}+z=f \cos \nu \tau+a_{0}\left(\frac{1}{(1-z)^{2}}-\frac{1}{(1+z)^{2}}\right)
$$

où $z=\frac{\delta_{\mathrm{a}}}{\lambda}, c=\frac{\alpha}{m \omega_{0}}, \omega_{0}=\sqrt{\frac{k}{m}}, \nu=\frac{\omega}{\omega_{0}}, f=\frac{F}{\lambda m \omega_{0}^{2}}$, $a_{0}=\frac{C_{1}}{\lambda^{3} m \omega_{0}^{2}}$ et $\tau=\omega_{0} t$ où $m$ représente la masse, $\alpha$ l'amortissement, $k$ la raideur, $F$ et $\omega$ représentent, respectivement, l'amplitude et la fréquence de l'excitation extérieure. La différentiation dans l'équation (3) est considérée par rapport au temps adimensionné $\tau$. Dans cette étude le but n'est pas d'avoir un modèle équivalent à l'expérimentation, mais d'observer les phénomènes élémentaires de sauts d'amplitude, les valeurs numériques utilisées ne sont donc pas recalées.

Comme l'expression du second membre de l'équation (3) n'est pas adaptée pour effectuer un traitement analytique direct, il est commode d'utiliser un développement en série de Taylor tronqué à l'ordre 3 :

$$
\left[\frac{1}{(1-z)^{2}}-\frac{1}{(1+z)^{2}}\right] \cong 4 z+8 z^{3}
$$

Cette expression est valable pour des petites valeurs de $z$, ce qui est le cas pour le problème considéré. Par conséquent, l'équation (3) prend la forme :

$$
z^{\prime \prime}+c z^{\prime}+\Omega_{0}^{2} z-\gamma z^{3}=f \cos \nu \tau
$$

où $\Omega_{0}^{2}=1-4 a_{0}$ et $\gamma=8 a_{0}$. 
Les méthodes de perturbation peuvent être utilisées pour étudier la dynamique de cet oscillateur du type Duffing à excitation extérieure. Pour analyser les solutions périodiques de l'équation (5) au voisinage de la résonance principale, un paramètre de perturbation $\varepsilon$ est introduit dans le terme non-linéaire de l'équation (5) et on impose la condition de la résonance principale sous la forme :

$$
\Omega_{0}^{2}=\nu^{2}+\varepsilon \sigma
$$

où $\sigma$ représente le paramètre mesurant l'écart par rapport à la résonance exacte.

En utilisant la méthode classique des échelles multiples [2], la solution périodique de l'équation (5) peut être approximée sous la forme d'un développement à deux échelles de temps de la forme :

$$
z(\tau)=z_{0}\left(\tau_{0}, \tau_{1}\right)+\varepsilon z_{1}\left(\tau_{0}, \tau_{1}\right)+\mathrm{O}\left(\varepsilon^{2}\right)
$$

où $\tau_{i}=\varepsilon^{i} \tau$. En termes des variables $\tau_{i}$, les dérivées temporelles deviennent : $\frac{\mathrm{d}}{\mathrm{d} t}=D_{0}+\varepsilon D_{0}+\mathrm{O}\left(\varepsilon^{2}\right)$ et $\frac{\mathrm{d}^{2}}{\mathrm{~d} t^{2}}=D_{0}^{2}+2 \varepsilon D_{0} D_{1}+\mathrm{O}\left(\varepsilon^{2}\right)$ avec $D_{0}=\frac{\partial}{\partial \tau}, D_{1}=\frac{\partial}{\partial \tau_{1}}$ et $D_{2}=\frac{\partial}{\partial \tau_{2}}$.

En suivant la procédure classique en substituant (6) et (7) dans (5), en égalant les coefficients de la même puissance en $\varepsilon$ et en éliminant les termes séculaires, le système de modulation d'amplitude $r$ et de phase $\theta$ (de la réponse) suivant est obtenu :

$$
\begin{aligned}
& \frac{\mathrm{d} r}{\mathrm{~d} t}=A_{1} r+H_{1} \sin \theta+H_{2} \cos \theta \\
& r \frac{\mathrm{d} \theta}{\mathrm{d} t}=B_{1} r+B_{2} r^{3}+H_{1} \cos \theta-H_{2} \sin \theta
\end{aligned}
$$

où $A_{1}=\frac{-c}{2}, H_{1}=\left(\frac{f \sigma}{8 \nu^{3}}-\frac{f}{2 \nu}\right), H_{2}=\frac{f c}{2 \nu^{2}}, B_{1}=\frac{\sigma}{2 \nu}-$ $\frac{3 c^{2} \nu^{2}+3 \sigma^{2}}{24 \nu^{3}}$ et $B_{2}=-\frac{5 \gamma}{12 \nu}$.

Après élimination de la phase $\theta$ du système (8), la réponse amplitude-fréquence s'écrit :

$$
B_{2}^{2} r^{6}+2 B_{1} B_{2} r^{4}+\left(A_{1}^{2}+B_{1}^{2}\right) r^{2}-\left(H_{1}^{2}+H_{2}^{2}\right)=0
$$

Cette équation algébrique d'ordre 3 en $r^{2}$ peut avoir une ou trois racines réelles. Les courbes de réponse données par l'équation (9) sont illustrées sur la figure 4 pour les valeurs des paramètres fixés $c=0,005, f=0,1$ et pour les différentes valeurs de $a_{0}[0,0001,0,006,0,01]$. Ces courbes de réponse montrent que le comportement du système considéré est de type assouplissant. Ce comportement se traduit par le glissement de la valeur de la fréquence de résonance vers des valeurs plus faibles. De plus, si le paramètre $a_{0}$, proportionnel au courant induit par les AEM est augmenté, le caractère assouplissant de la réponse augmente aussi. Pour de très faibles valeurs de $a_{0}$ tendant vers zéro, le cas linéaire est bien retrouvé. La figure 4 montre aussi que pour des grandes valeurs de $a_{0}$ la courbe de résonance glisse vers des valeurs des fréquences plus faibles. Pour valider ces résultats analytiques (lignes continues pour les solutions stables et discontinues pour la solution instable), des simulations numériques ont été effectuées directement sur l'équation (5) en utilisant la méthode de Runge-Kutta (cercles dans Fig. 4).
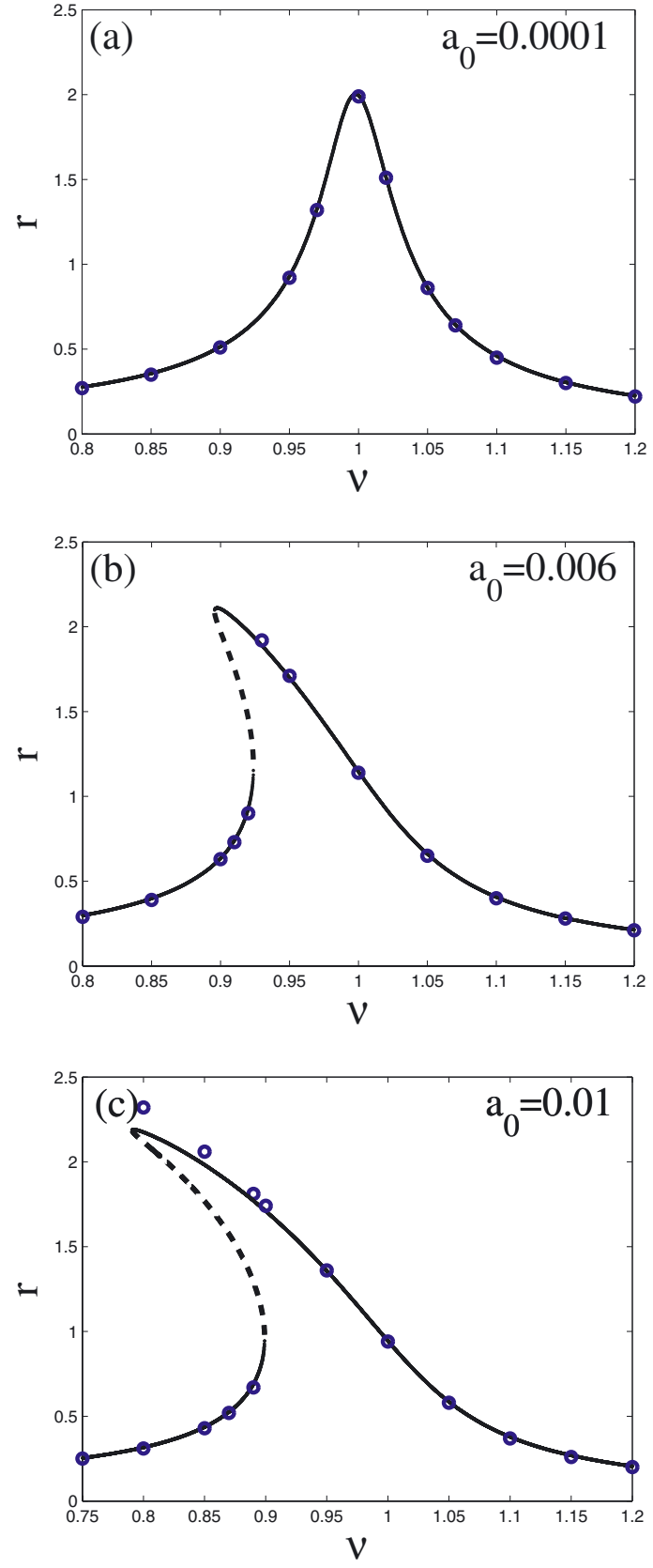

Fig. 4. Effet de $a_{0}$ sur la courbe de réponse au voisinage de la résonance principale; $c=0,05$ et $f=0,1$. Solutions stables (ligne continue), solution instable (ligne discontinue), intégration numérique (cercles).

\section{4 Étude éléments-finis}

Cette étape d'investigation numérique est essentielle afin d'évaluer l'influence des paramètres des actionneurs (courant et entrefer) sur le comportement de la structure et la possibilité d'introduire des phénomènes d'hystérésis. Cette étude fait suite au traitement analytique réalisé sur un système à un seul degré de liberté montrant les phénomènes de base. Ici, une étude éléments-finis dans des conditions proches de l'expérimentation est effectuée. 
Le bâti et l'encastrement sont modélisés par des masses infiniment rigides. La poutre (masse de $610 \mathrm{~g}$ ) est modélisée par 20 éléments de poutre de Timoshenko avec 5 degrés de liberté par nœud, 3 déplacements et 2 rotations (suivant les directions $X$ et $Z$ ). L'ensemble I de l'actionneur est fixé sur la poutre et contribue, dans la gamme fréquentielle de l'étude, uniquement par sa masse (282 g). L'équation générale qui régit le comportement s'écrit classiquement [10] par :

$$
M \ddot{\delta}+C \dot{\delta}+K \delta=F_{\text {extérieure }}+F_{\text {em }}
$$

où $M, C$ et $K$ représentent, respectivement, les matrices de masse, d'amortissement visqueux et de raideur. $\delta$ est le déplacement et $F_{\text {extérieure }}$ est l'excitation externe. L'effet de l'AEM est pris en compte dans le second membre avec la force $F_{\text {em }}$.

La résolution temporelle est effectuée en utilisant un algorithme de type Runge-Kutta d'ordre 4 dans l'environnement Matlab ${ }^{\circledR}$ et SIMULINK ${ }^{\circledR}$. L'équation (9) mise sous forme d'état peut s'écrire :

$$
\begin{aligned}
& \dot{X}=A X+B U \\
& {\left[\begin{array}{l}
\dot{\delta} \\
\ddot{\delta}
\end{array}\right]=\left[\begin{array}{lc}
0 & I \\
-M^{-1} \cdot K & -M^{-1} \cdot C
\end{array}\right]\left[\begin{array}{l}
\delta \\
\dot{\delta}
\end{array}\right]+\left[\begin{array}{l}
0 \\
M^{-1}
\end{array}\right] } \\
& \times\left(F_{\text {extérieure }}+F_{\mathrm{em}}\right)
\end{aligned}
$$

où $X$ est le vecteur d'état, $A$ est la matrice d'évolution d'état et $B$ est la matrice de commande.

L'objectif des simulations est d'observer le comportement dynamique autour de la première fréquence propre du système. Les calculs sont réalisés à partir d'un modèle de la structure souple obtenue par réduction modale sur les huit premiers modes. Un facteur d'amortissement visqueux modal de 0,005 est utilisé pour tous les modes, cette valeur est proche de la valeur identifiée expérimentalement. Les simulations numériques sont réalisées sous l'environnement Matlab ${ }^{\circledR}$ et Simulink ${ }^{\circledR}$. La réponse de la structure (en temps réel) est calculée au nœud \#7 (position du capteur de déplacement). La force extérieure est un balayage sinus d'amplitude $0,1 \mathrm{~N}$ et dont la fréquence décroît de $5 \mathrm{~Hz}$ au-dessus de la première fréquence de résonance à $5 \mathrm{~Hz}$ au-dessous. Le balayage est discret à progression linéaire afin d'avoir des conditions de simulations les plus proches de l'expérimentation. La durée de simulations est de $200 \mathrm{~s}$.

Les réponses obtenues, pour une configuration de $1 \mathrm{~mm}$ d'entrefer et un courant de $1 \mathrm{~A}$, sont présentées sur la figure 5. Le phénomène de saut d'amplitude est observé clairement en balayage croissant ou décroissant. Le comportement assouplissant est observé également, il est à noter que le glissement de la fréquence de résonance en balayage décroissant est plus important. L'ampleur du phénomène de saut peut être contrôlée en fonction des deux paramètres entrefer et courant.

Tout d'abord, la réponse de la structure est calculée pour un courant constant de 1 A et pour différentes

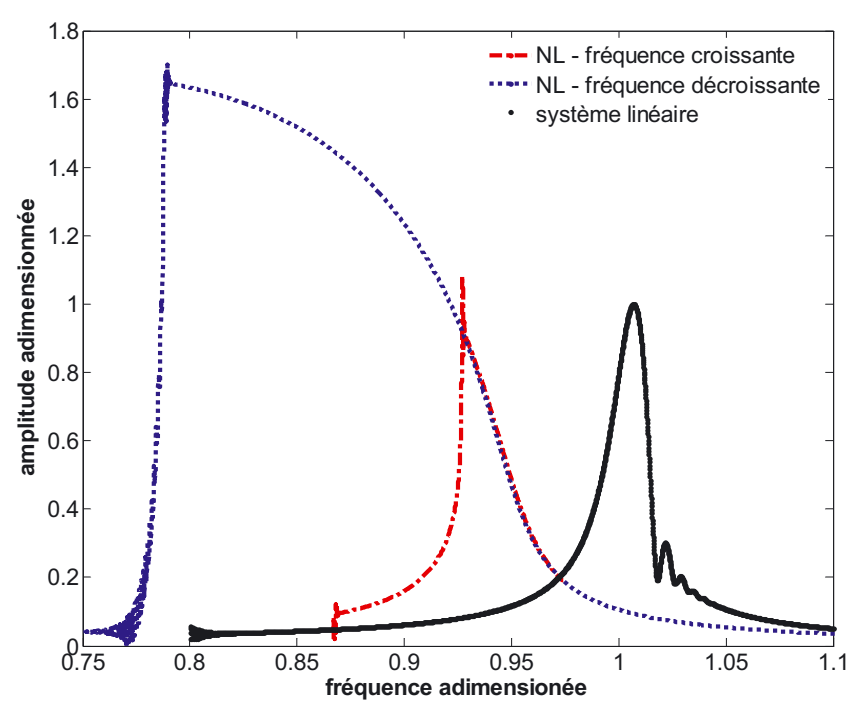

Fig. 5. Réponse de la structure pour un entrefer de $1 \mathrm{~mm}$ et un courant de $1 \mathrm{~A}$.

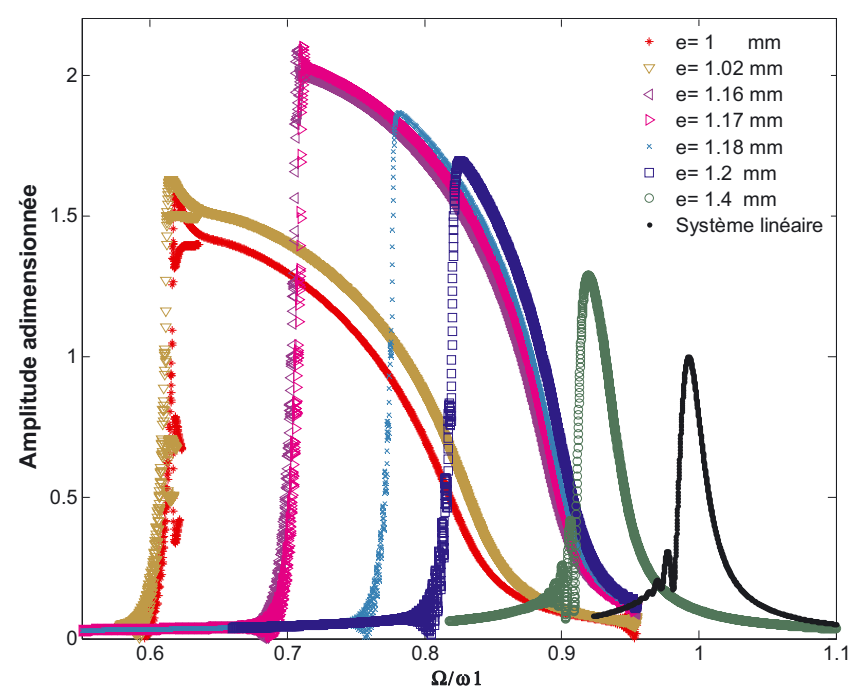

Fig. 6. Influence de la variation de l'entrefer sur la réponse de la structure (courant constant de $1 \mathrm{~A}$ ).

valeurs d'entrefer (Fig. 6). Pour des valeurs d'entrefer relativement petites (1 et 1,02 $\mathrm{mm}$ ), le phénomène de saut d'amplitude peut être observé très nettement avec un comportement très instable. Au fur et à mesure que l'entrefer augmente, cette instabilité diminue mais le phénomène de saut d'amplitude est toujours observable. Pour des valeurs importantes d'entrefer $(1,4 \mathrm{~mm})$, le phénomène de saut d'amplitude n'est plus tellement marqué, par contre, le glissement fréquentiel du pic de résonance vers de plus faibles valeurs fréquentielles subsiste : assouplissement.

Ensuite, la réponse de la structure est calculée pour un entrefer constant de 1,2 mm et pour différentes valeurs de courant (Fig. 7). Ici, plus le courant est important plus le phénomène de saut est marqué joint à un comportement instable et au phénomène d'assouplissement. 


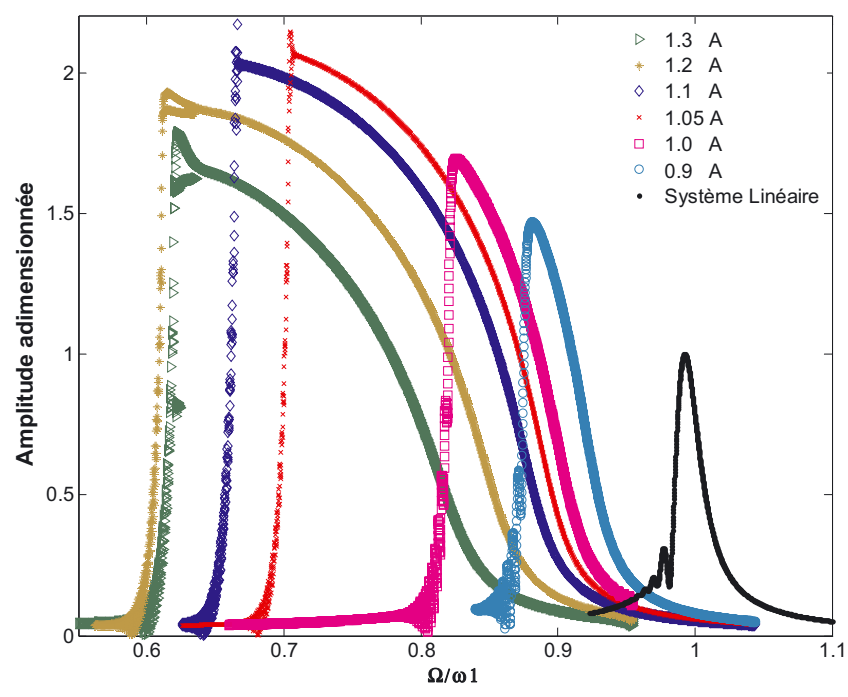

Fig. 7. Influence de la variation du courant sur la réponse de la structure (entrefer constant de 1,2 $\mathrm{mm}$ ).

Les résultats des simulations montrent donc clairement qu'il est possible d'avoir une structure à comportement non-linéaire doté d'hystérésis et dont les paramètres peuvent être pilotés par des actionneurs électromagnétiques.

Le traitement analytique effectué a mis en évidence, sur un modèle simplifié de la poutre encastrée libre, ce caractère assouplissant caractérisé par le phénomène de glissement de la courbe de résonance vers des valeurs plus faibles.

\section{5 Étude expérimentale}

Afin de valider les résultats obtenus précédemment, un balayage sinus (entre 5 et $20 \mathrm{~Hz}$ ) en utilisant le pot d'excitation (Fig. 1) est réalisé dans la configuration $1 \mathrm{~mm}$ d'entrefer et 0,5 A. Le positionnement de l'actionneur sur la poutre a été choisi pour que l'amplitude de la force d'excitation puisse être considérée comme constante pendant la durée des expériences.

Le déplacement est mesuré par un capteur sans contact de type Vibrometer TQ 102, l'excitation harmonique est appliquée en utilisant un pot électromagnétique du type B\&K 4810. Le pot d'excitation est placé sur une suspension souple et sa table vibrante est liée à la poutre en un point proche de l'encastrement afin d'éviter la modification de la dynamique de la structure étudiée. L'acquisition et le traitement des signaux sont effectués à l'aide d'un analyseur de spectre de type Agilent 35670A.

Les déplacements mesurés en fonction d'un balayage sinus (croissant ou décroissant) de la force d'excitation montrent la présence de saut d'amplitude dû au phénomène d'hystérésis (Fig. 8). Il est à noter que les actionneurs ont un effet assouplissant sur le comportement dynamique de la structure dans cette gamme fréquentielle. Aussi, le caractère de glissement de la

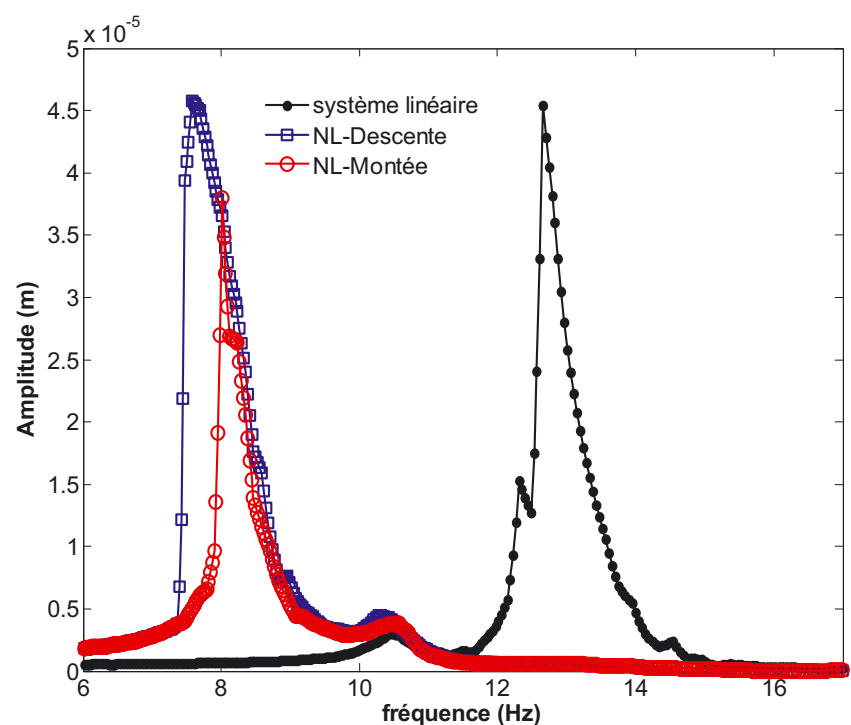

Fig. 8. Déplacements mesurés en fonction d'un balayage croissant et décroissant de l'excitation, $e=1 \mathrm{~mm}, I=0,5 \mathrm{~A}$.

réponse vers des faibles valeurs de fréquences est retrouvé expérimentalement. Plusieurs répétitions de mesures sont réalisées montrant que la structure se comporte d'une façon identique. L'effet du régime transitoire a été également étudié, ici seuls les résultats en régime permanent sont présentés : balayage lent de 5 à $20 \mathrm{~Hz}$ en $200 \mathrm{~s}$.

\section{Conclusions}

L'étude présentée dans ce travail concerne la conception et la réalisation d'une structure à comportement non-linéaire dont la non-linéarité est introduite par des AEM. L'objectif principal est de mettre en évidence les phénomènes d'hystérésis responsables de saut d'amplitude dans les réponses fréquentielles d'une poutre encastrée libre excitée harmoniquement et de montrer l'influence de la force électromagnétique sur la courbe de réponse et sur l'hystérésis.

Les résultats analytiques, numériques et expérimentaux obtenus montrent qu'il est possible de piloter ces phénomènes de saut d'amplitude dans une structure flexible élancée en faisant varier les caractéristiques des AEM. Les différentes techniques d'analyse ont confirmé que les actionneurs ont un effet mollissant sur le comportement dynamique de la structure considérée pour la gamme fréquentielle étudiée. Il a été montré aussi que les AEM causent un glissement de la courbe de réponse vers de faibles valeurs des fréquences.

Les résultats de cette étude ouvrent des perspectives multiples dans le domaine du contrôle actif de la dynamique non-linéaire des structures flexibles. L'utilisation des actionneurs électromagnétiques permet de modifier la fréquence propre de la structure. Cette propriété peut être utilisée pour qu'une structure, soumise à une excitation harmonique variable et connue, ne soit jamais excitée sur une de ses fréquences propres. 


\section{Références}

[1] L.N. Virgin, Introduction to experimental nonlinear dynamics, Cambridge University Press, 2000, p. 256

[2] A.H. Nayfeh, D.T. Mook, Nonlinear Oscillations, Wiley, New York, 1979

[3] G. Michon, L. Manin, R.G. Parker, R. Dufour, Duffing oscillator with parametric excitation: analytical and experimental investigation on a belt-pulley system, ASME J. Comput. Nonlinear Dyn. 3 (2008) 031001

[4] M. Belhaq, A. Fahsi, $2: 1$ and $1: 1$ frequency-locking in fast excited van der Pol-Mathieu-Duffing oscillator, Nonlinear Dyn. 53 (2008) 139-152

[5] A. Fahsi, M. Belhaq, F. Lakrad, Suppression of hysteresis in a forced van der Pol-Duffing oscillator, Commun. Nonlinear Sci. Numer. Simul. 14 (2009) 2426-2433

[6] G. Schweitzer, H. Bleuler, A. Traxler, Active Magnetic Bearings - Basics, Properties and Applications, vdf Hochschulverlag AG, ETH, Zurich, 2003
[7] A. El-Shafei, A.S. Dimitri, Controlling Journal bearing instability using active magnetic bearings, Proc. ASME Turbo Expo, GT2007-28059, Canada, 2007

[8] Y. Skladanek, J. Der Hagopian, J. Mahfoud, Energy Cost of the Active Control of Rotating Machine by Using Electromagnetic Actuator and Piezoelectric Actuator, ASME Gas Turbine Technical Congress \& Exposition, June 8-12, 2009, Orlando, Florida, USA

[9] J. Der Hagopian, J. Mahfoud, Numerical and Experimental Investigation of the Active Control by Using Electromagnetic Actuator, Proc. XIII International Symposium on Dynamic Problems of Mechanics (DINAME 2009), C.A. Almeida, (Ed.), ABCM, Angra dos Reis, RJ, Brazil, March 2nd-6th, 2009

[10] M. Lalanne, P. Berthier, J. Der Hagopian, Mechanical Vibrations for Engineers, Wiley Interscience Publication, $2^{\mathrm{e}}$ édition, 1998, p. 266 